\title{
Endogenous post-transcriptional gene silencing of flavone synthase resulting in high accumulation of anthocyanins in black dahlia cultivars.
}

\section{$\operatorname{AUTHOR(S):~}$}

Deguchi, Ayumi; Ohno, Sho; Hosokawa, Munetaka; Tatsuzawa, Fumi; Doi, Motoaki

\section{CITATION:}

Deguchi, Ayumi ...[et al]. Endogenous post-transcriptional gene silencing of flavone synthase resulting in high accumulation of anthocyanins in black dahlia cultivars.. Planta 2013, 237(5): 1325-1335

\section{ISSUE DATE:}

2013-05

URL:

http://hdl.handle.net/2433/189857

\section{RIGHT:}

The final publication is available at Springer via http://dx.doi.org/10.1007/s00425-0131848-6; This is not the published version. Please cite only the published version.; この論文 は出版社版でありません。引用の際には出版社版をご確認ご利用ください。 
Endogenous post-transcriptional gene silencing of flavone synthase resulting in high accumulation of anthocyanins in black dahlia cultivars

Ayumi Deguchi ${ }^{1} \cdot$ Sho Ohno $^{1 *} \cdot$ Munetaka Hosokawa $^{1 *} \cdot$ Fumi Tatsuzawa $^{2} \cdot$ Motoaki Doi $^{1}$

${ }^{1}$ Graduate School of Agriculture, Kyoto University, Sakyo-ku, Kyoto 606-8502, Japan

${ }^{2}$ Faculty of Agriculture, Iwate University, Morioka 020-8550, Japan

*Corresponding author: Sho Ohno

Laboratory of Vegetable and Ornamental Horticulture, Graduate School of Agriculture, Kyoto University, Sakyo-ku, Kyoto 606-8502, Japan

Telephone: $+81-75-753-6048$

Fax: +81-75-753-6068

E-mail: ono.sho.47n@st.kyoto-u.ac.jp

*Corresponding author: Munetaka Hosokawa

Laboratory of Vegetable and Ornamental Horticulture, Graduate School of Agriculture, Kyoto University, Sakyo-ku, Kyoto 606-8502, Japan

Telephone: $+81-75-753-6048$

Fax: +81-75-753-6068

E-mail: mune@kais.kyoto-u.ac.jp 


\begin{abstract}
Black color in flowers is a highly attractive trait in the floricultural industry, but its underlying mechanisms are largely unknown. This study was performed to identify the bases of the high accumulation of anthocyanidins in black cultivars and to determine whether the high accumulation of total anthocyanidins alone leads to the black appearance. Our approach was to compare black dahlia (Dahlia variabilis) cultivars with purple cultivars and a purple flowering mutant of a black cultivar, using pigment and molecular analyses. Black cultivars characteristically exhibited low lightness, high petal accumulation of cyanidin and total anthocyanidins without flavones, and marked suppression of flavone synthase $(D v F N S)$ expression. A comparative study using black and purple cultivars revealed that neither the absence of flavones nor high accumulation of total anthocyanidins is solely sufficient for black appearance, but that cyanidin content in petals is also an important factor in the phenotype. A study comparing the black cultivar 'Kokucho' and its purple mutant showed that suppression of DvFNS abolishes the competition between anthocyanidin and flavone synthesis and leads to accumulation of cyanidin and total anthocyanidins that produce a black appearance. Surprisingly, in black cultivars the suppression of $D v F N S$ occurred in a post-transcriptional manner, as determined by small RNA mapping.
\end{abstract}

\title{
Key words:
}

Black flower, Cyanidin, Dahlia variabilis, Flavone synthase, Post-transcriptional gene silencing, Short interfering RNA

$\begin{array}{ll}\text { Abbreviations } & \\ \text { ANS } & \text { Anthocyanidin synthase } \\ \text { bHLH } & \text { Basic helix-loop-helix } \\ \text { CHI } & \text { Chalcone isomerase } \\ \text { CHS } & \text { Chalcone synthase } \\ \text { DFR } & \text { Dihydroflavonol 4-reductase } \\ \text { F3H } & \text { Flavanone 3-hydroxylase } \\ \text { F3'H } & \text { Flavonoid 3'-hydroxylase } \\ \text { FLS } & \text { Flavonol synthase } \\ \text { FNS } & \text { Flavone synthase } \\ \text { GST } & \text { Glutathione S-transferase } \\ \text { GT } & \text { Glucosyltransferase }\end{array}$


HPLC

MT

PTGS

RACE

siRNA
High performance liquid chromatography

Malonyltransferase

Post-transcriptional gene silencing

Rapid amplified cDNA end

Short interfering RNA

\section{Introduction}

Dahlia (Dahlia variabilis) has huge variation in flower traits, owing to its high polyploidy. It belongs to the Asteraceae and is believed to be an autoallooctoploid (Gatt et al. 1998). About 50,000 dahlia cultivars were produced by breeders in the last century (McClaren 2009). Flower color variation in particular is enormous, and red, yellow, purple, pink, ivory, and black cultivars have been developed. Dahlia accumulates flavonoids including anthocyanins, butein (6'-deoxychalcone), and flavones as pigments in its petals (Harborne et al. 1990, Nordström and Swain 1953, Price 1939, Takeda et al. 1986).

The flavonoid biosynthetic pathway is the best studied plant secondary metabolite synthetic pathway, and the genes of the pathway enzymes have been isolated in many species (Tanaka et al. 2008). First, chalcone synthase (CHS) catalyzes the synthesis of naringenin chalcone from three molecules of malonyl CoA and one molecule of 4-coumaroyl CoA. Then anthocyanidins are synthesized via the action of chalcone isomerase (CHI), flavanone 3-hydroxylase $(\mathrm{F} 3 \mathrm{H})$, flavonoid 3'-hydroxylase (F3'H), dihydroflavonol 4- reductase (DFR), and anthocyanidin synthase (ANS). Flavones are synthesized by flavone synthase (FNS) from flavanones, the products of CHI. Anthocyanidins and flavones are finally subjected to modification by enzymes such as glucosyltransferase (GT) and malonyltransferase (MT) and transported to vacuoles by glutathione S-transferase (GST). Genes encoding transcriptional regulators of structural genes are also well studied and basic Helix-Loop-Helix (bHLH), MYB, and WD-repeat transcription factors regulate some structural genes alone or via a transcriptional complex (Hichri et al. 2011, Koes et al. 2005).

Flower color is one of the most important traits in horticultural crops, and black coloring in flowers is an especially attractive trait but one present in few genera. The mechanism for black coloring has not been determined, so that breeding black cultivars depends on crossing. Generally, deep coloring is caused by a high accumulation of pigments. For example, in grape species (Vitis $\times$ labruscana and $V$. vinifera), berry color is determined by the amount of anthocyanins in berry skins, and this amount is affected by MYB A genes (Azuma et al. 2011, Kobayashi et al. 2002, Kobayashi et al. 2005, Kobayashi 2009). However, other factors such as cellular architecture and pH also affect flower color (Grotewold 2006), so the molecular mechanism for black appearance remains to be elucidated.

In dahlia, the genetics of flower color inheritance was studied long ago (Lawrence 1929, 
Lawrence 1931, Lawrence and Scott-Honcrieff 1935), and some genes for anthocyanin synthesis have been isolated. A bHLH transcription factor, DvIVS belonging to the ANI subgroup in Petunia hybrida (Spelt et al. 2000) and TT8 in Arabidopsis thaliana (Nesi et al. 2000), is involved in anthocyanin synthesis via the regulation of $D v C H S 1, D v F 3 H, D v D F R$, and $D v A N S$ (Ohno et al. 2011a), and a positive correlation between anthocyanin amount and DvIVS expression was observed in cyanic cultivars (Ohno et al., submitted for publication).

In previous studies, black dahlia cultivars accumulated high amount of total anthocyanidins without flavone accumulation (Ohno et al. 2011b, Thill et al. 2012), but this accumulation was not explained by DvIVS expression levels. It was accordingly suggested that another mechanism should underlie the black appearance. This study was performed to identify the basis of high total anthocyanidin accumulation and determine whether high accumulation of total anthocyanidins truly accounts for black appearance. We compared black cultivars with purple cultivars and with a purple flowering mutant of a black cultivar, using pigment and molecular analyses. Based on the results, we present a model explaining black color appearance in dahlia.

\section{Materials and Methods}

Plant materials

The black cultivars 'Fidalgo Blacky', 'Ms. Noir', 'Kokucho', 'Black Cat', and 'Kokucho' mutant and the purple cultivars 'Cupid', 'Yukino', 'Super Girl', 'Atom', and 'Evelyn Rumbold' were used (Fig. 1). The 'Kokucho' mutant, which produces purple flowers, was obtained as a spontaneous lateral mutant in a commercial field of a Japanese dahlia grower, Yukihiro Fukuda. All cultivars were grown on the experimental farm of Kyoto University.

For analysis of temporal pigment accumulation and gene expression, petal development was divided into four stages: stage 1: uncolored, stage 2 : half colored, stage 3 : completely colored and unexpanded, stage 4: completely colored and expanded.

Color analysis

For objective flower color evaluation, color components of the CIE L*a*b* coordinate, namely lightness $\left(L^{*}\right)$ and chroma $\left(c^{*}\right.$ : calculated as $\left.c^{*}=\left(a^{* 2}+b^{* 2}\right)^{1 / 2}\right)$ were measured with a hand spectrophotometer (NR-3000, Nippon Denshoku Industries Co., Ltd., Tokyo, Japan). Three areas of the adaxial surface were subjected to color measurement. Scores of three replicate petals at stage 4 from three different flowers 
were averaged.

Composition and quantification of pigments

Quantification of pigments was performed by high-performance liquid chromatography (HPLC). One hundred grams of fresh petals were homogenized with a mortar and pestle under liquid nitrogen. Then 1 $\mathrm{ml}$ of extraction solution (acetic acid:methanol:water 1:4:5, v/v/v) was added, and the mixture was stored overnight at $4{ }^{\circ} \mathrm{C}$ in the dark. The mixture was centrifuged at $4^{\circ} \mathrm{C}$ at $15,000 \mathrm{rpm}$ for $15 \mathrm{~min}$, and the supernatant was collected into a Petri dish and dried completely. Dried pigments were redissolved in $1 \mathrm{ml}$ of $10 \%$ hydrochloric acid in $50 \%$ methanol. After visual confirmation of the absence of a precipitate, the solution was diluted 20 times with the same solvent. For hydrolysis, $500 \mu \mathrm{l}$ of diluted solution was boiled at $95^{\circ} \mathrm{C}$ for 1 hour and $20 \mu \mathrm{l}$ was injected into the HPLC. HPLC analysis was performed according to Ohno et al. (2011a).

To make a standard curve, $1 \mathrm{mg}$ of pelargonidin (extracted by thin layer chromatography), cyanidin chloride (Wako, Kyoto, Japan), apigenin (Wako), and luteolin (LKT Laboratories Inc., St. Paul, MN, USA) were eluted in $600 \mu \mathrm{l}$ of $10 \%$ hydrochloric acid in 50\% methanol, and a one-half dilution series was made. Twenty micro liters of each dilution was injected into the HPLC and pigment amounts were calculated from peak areas. Calculations for cyanidin were converted from those for cyanidin chloride.

RNA and DNA extraction

Total RNA was extracted using Sepasol ${ }^{\circledR}$-RNA I Super G (Nacalai tesque, Kyoto, Japan) with purification by High-Salt Solution for Precipitation (Takara Bio Inc., Ohtsu, Japan) or Get pure RNA Kit (Dojindo, Kumamoto, Japan). Genomic DNA was extracted with MagExtractor ${ }^{\mathrm{TM}}$-Plant Genome (Toyobo Co. Ltd., Osaka, Japan).

Isolation of $D v F N S$ and $D v G S T$

A partial sequence of $D v F N S$ was previously isolated using degenerate primers (Ohno et al. 2011c), and a partial sequence of $D v G S T$ was isolated by rapid amplified cDNA end (RACE) of $D v W D R 2$ (Ohno et al. 2011a). The 3' and 5' RACE using a RACE library constructed with GeneRacer ${ }^{\mathrm{TM}}$ Kit (Invitrogen, Carlsbad, CA, USA) of 'Michael J' (Ohno et al. 2011a) were performed. Primers for full-length cDNAs and for real-time RT-PCR were designed (Table S1). Sequencing analysis was performed using a BigDye $^{\circledR}$ Terminator v 3.1 Cycle Sequencing Kit and a 3100 Genetic Analyzer (Applied Biosystems, Foster City, CA, USA). 
Real-time RT-PCR

Expression analysis of $D v C H S 1, D v C H S 2, D v C H I, D v F 3 H, D v F 3$ 'H, DvDFR, DvANS, Dv3GT, Dv3MT, $D v G S T, D v F N S$, and $D v I V S$ was performed by real-time RT-PCR. Total RNA was reverse transcribed with ReverTra Ace (Toyobo), and real-time RT-PCR was performed with SYBR Premix Ex TaqII (Takara) using a 7900HT Fast Real-Time PCR System (Applied Biosystems). Real-time RT-PCR was performed as follows: $95^{\circ} \mathrm{C}$ for $10 \mathrm{~s}$ followed by 40 cycles of $95^{\circ} \mathrm{C}$ for $10 \mathrm{~s}$ and $60^{\circ} \mathrm{C}$ for $30 \mathrm{~s}$; single-target product amplification was checked using dissociation curves. DvActin was used as an internal standard. Primers for $D v 3 M T, D v G S T, D v F 3^{\prime} H$ and $D v F N S$ are given in Table S1, primers for other genes were given in Ohno et al. (2011c).

Detection of siRNAs

The detection of short interfering RNA (siRNA) of DvFNS was performed according to Ohno et al. (2011c). A digoxigenin-labeled RNA probe was synthesized using T7 RNA polymerase (F. Hoffmann-La Roche AG, Basel, Switzerland) by in vitro transcription of a 3'-digested pTAC-1 vector (BioDynamics Laboratory Inc., Tokyo, Japan) carrying the full length of $D v F N S$ in the antisense orientation.

Mapping of small RNAs

For deep sequencing of small RNAs, RNAs abundant in small RNAs were extracted from $200 \mathrm{mg}$ of stage 2 fresh petals of 'Kokucho' using a MirVana miRNA Isolation Kit (Applied Biosystems) and sequenced by a 100-bp paired-end method using an Illumina HiSeq 2000 (Illumina Inc., San Diego, CA, USA). Small RNAs (18-32 nt long) from read 1 were mapped onto the DvFNS genome as a reference sequence, with no mismatches allowed.

Statistical analysis

Significant differences in pigment amounts between 'Kokucho', and the mutant were assessed by Mann-Whitney $U$ test $(P<0.05)$. All analyses were performed with Microsoft Excel.

\section{Results}


Color differences

To evaluate petal colors, color differences were measured and expressed on the $\mathrm{L}^{*} \mathrm{C}^{*}$ coordinate. $\mathrm{L}^{*}$ indicates the lightness of the color, so that low lightness contributes to a black and high lightness contributes to a white appearance. In contrast, $c^{*}$ indicates chroma calculated from $a^{*}$ and $b^{*}$. Positive $a^{*}$ and $b^{*}$ values indicate magenta and yellow, while negative values indicate green and blue. Black cultivars were located at lower $\mathrm{L}^{*}$ values than purple cultivars (Fig. 2). Among black cultivars, the $\mathrm{c}^{*}$ value tends to decrease with the $\mathrm{L}^{*}$ value. The $\mathrm{c}^{*}$ values could not distinguish black from purple cultivars. In black cultivars, 'Fidalgo Blacky', which has the blackest appearance, showed the lowest $\mathrm{L}^{*}$, followed by 'Ms. Noir' and 'Kokucho'. Thus the appearance of blackish flower color corresponded to the $\mathrm{L}^{*}$ value. Purple cultivars exhibiting deep color, also showed lower $\mathrm{L}^{*}$ values, and paler cultivars showed higher $\mathrm{L}^{*}$ values.

Pigment analyses

All black cultivars accumulated only anthocyanidins, whereas all purple cultivars accumulated anthocyanidins and flavones (Fig. 3). The total anthocyanidin content in all black cultivars was at least $1.00 \mathrm{mg}$ per $100 \mathrm{mg}$ fresh petals, whereas that of purple cultivars 'Cupid', 'Evelyn Rumbold', and 'Atom' was lower than $1.00 \mathrm{mg}$ per $100 \mathrm{mg}$ (Fig. 3). Although higher accumulation of total anthocyanidins was detected in 'Super Girl' and 'Yukino' than in 'Fidalgo Blacky' and 'Black Cat' (Fig. 3), they did not appear black but purple, indicating that total anthocyanidin amounts could not be the only factor determining black appearance. For explaining the appearance of black color, the amount of cyanidin seemed to be important, as discussed later.

The mutant produced purple flowers, whereas the original flower color of 'Kokucho' was black. The value of $L^{*}$ in the mutant was higher than that of the original and was similar to those of purple cultivars (Fig. 2). The mutant accumulated flavones in addition to lower amounts of anthocyanidins than the original (Fig. 4A, B), indicating that the phenotype of this mutant is similar to that of purple cultivars. Distinct accumulation of anthocyanidins started at developmental stage 3 in both lines, whereas accumulation of flavones started in an early stage in the mutant (Fig. 4A, B). Comparison of pigment amounts in stage 3 between the original and the mutant showed no significant difference in pelargonidin but significant differences in cyanidin, apigenin, and luteolin (Fig. 4C).

\section{Real-time RT-PCR}

With the aim of determining the regulation of flavonoid biosynthetic genes in black cultivars, genes for flavone synthase and glutathione S-transferase were first isolated. The full length of the DvFNS cDNA 
coding region in 'Kokucho' (AB769842) was 1545 bp and it encoded 514 amino acid residues. This deduced protein shares $70 \%$ identity with flavone synthase II of Gerbera hybrid cultivar (AAD39549) (Martens and Forkmann 1999), 56\% identity with flavone synthase II of Glycine max (ACV65037) (Fliegmann et al. 2010), and 54\% identity with flavone synthase II of Gentiana triflora (BAD91809) (Nakatsuka et al. 2005), and was completely identical with FNS in Dahlia variabilis 'Feuerschein' (ADM67335) (Thill et al. 2012). The full length of the DvGST cDNA coding region in 'Michael J' (AB769840) was $651 \mathrm{bp}$ and encoded 216 amino acid residues. This deduced protein shares 67\% identity with Petunia hybrida AN9 (CAA68993) (Mueller et al. 2000) and 51\% identity with Arabidopsis thaliana TRANSPARENT TESTA 19 (Q9FE46) (Kitamura et al. 2004), proteins reported to be involved in anthocyanin accumulation.

To determine whether these genes play an important role in black color expression, real-time RT-PCR was performed for genes in the flavonoid biosynthetic pathway, including the newly isolated genes. In comparison to purple cultivars, all genes were expressed less highly in black cultivars (Fig. S1), but DvFNS showed significantly lower expression in black cultivars (Fig. 5). This observation was consistent with a previous report that flavone synthase protein activity is low in black cultivars (Thill et al. 2012). To characterize the temporal expression patterns, petals of stages 1-3 were examined. Highest $D v F N S$ expression was observed in stage 2 in purple cultivars, while little or no expression was detected in any stage in black cultivars (Fig. S2). This early expression pattern was consistent with previous reports in other genera (Akashi et al. 1999, Martens and Forkmann 1998, Nakatsuka et al. 2005). In the comparison between 'Kokucho' original and the mutant, the same tendency was detected, with only DvFNS being expressed in the mutant (Fig. 5).

Analysis of DvFNS small RNAs

To determine whether the low expression of $D v F N S$ in black cultivars was due to PTGS, DvFNS siRNA detection was performed using RNA gel blot analysis. In black cultivars, siRNAs were detected in abundance in 'Kokucho' and 'Black Cat' and at low levels in 'Fidalgo Blacky' and 'Ms. Noir', whereas none were detected in purple cultivars or the 'Kokucho' mutant (Fig. 6). The suppression of DvFNS corresponded to the detection of DvFNS siRNA, suggesting that suppression was induced post-transcriptionally. To confirm that DvFNS was post-transcriptionally silenced, small RNAs were mapped to the DvFNS genome. As reference sequence, genomic sequences of DvFNS in 'Kokucho' original (AB769481) and the mutant were used. The DvFNS coding region contained one intron of 1,150 bp, and the sequence was completely identical between 'Kokucho' original and the mutant. Of the $14,895,667$ reads, 9,368,245 were 18-32 nt long and 11,574 of these short reads were mapped onto the DvFNS genome. The 21-nt mapped small RNAs accounted for 10,279 reads and were most mapped onto exon 2 (Fig. 7). Distinct 21-nt antisense small RNAs numbered 4,192 and mapped to position 2307-2327 
of the DvFNS genome. These observations demonstrate that $D v F N S$ was silenced post-transcriptionally by siRNAs in black cultivars.

\section{Discussion}

Phenotypic characters in black cultivars

Despite the "black" designation of so-called black cultivars ('Kokucho' means "black butterfly"), the evaluation of the appearance is subjective. For this reason, color differences were measured objectively. Whereas the $\mathrm{c}^{*}$ value could not distinguish black and purple cultivars, the $\mathrm{L}^{*}$ value was markedly lower in black cultivars (Fig. 2). $\mathrm{L}^{*}$ indicates lightness of the color, such that higher lightness means whiter and lower lightness means blacker. Accordingly, a lower lightness than that of purple cultivars makes cultivars black. The orders from visual blackness and that from $L^{*}$ value were the same, suggesting that lower $\mathrm{L}^{*}$ value can be used as an indicator for black appearance.

Cyanidin as an important factor in black appearance

The results of flavonoid quantification suggested the following responsible factors for black appearance.

\section{Absence of flavones}

Flavones were detected in purple but not in black cultivars (Fig. 3). Given that yellow, red, purple, pink, and ivory cultivars generally accumulate flavones (Ohno et al. 2011a, Ohno et al. 2011c, Ohno et al. submitted for publication), the absence of flavones is a characteristic common among almost-black cultivars. But this absence cannot be a sufficient condition for a black appearance, given that a certain pink cultivar does not accumulate flavones (Deguchi et al. unpublished data), and that some black cultivars accumulate flavones (Deguchi et al. unpublished data, Thill et al. 2012). This assertion is supported by the finding that gerbera plants with homologous recessive fns alleles accumulated higher anthocyanins than plants with the fns+ allele lacking flavones, but did not appear black (Martens and Mithöfer 2005). The relationship between the anthocyanin synthesis pathway and the flavone and flavonol pathways that branch halfway along it has been studied, and it is suggested that they are in competition (Davies et al. 2003). Because anthocyanins and flavones are synthesized through the same biosynthetic pathway, it may be expected that the absence of flavones contributes to high anthocyanin accumulation by abolishing this competition. 


\section{High accumulation of anthocyanidins}

A negative correlation was observed between $L^{*}$ value and total anthocyanidins $(r=-0.604)$, indicating that high total anthocyanidins contribute to a black appearance. Although it has been reported that high accumulations of total anthocyanidins are the cause of the black appearance (Ohno et al. 2011c, Thill et al. 2012), it is impossible to explain black appearance only by high total anthocyanidins. In the purple cultivars 'Super Girl' and 'Yukino', higher amounts of total anthocyanidins accumulated than in the black cultivars 'Fidalgo Blacky' and 'Black Cat' (Fig. 3). Although a certain amount is needed for black appearance, the high accumulation of total anthocyanidins alone cannot explain the difference between black and purple cultivars.

The expression of the anthocyanin synthesis regulatory factor DvIVS also could not explain the difference between black and purple cultivars (Fig. S1), suggesting that the high accumulation of total anthocyanidins is caused not by high expression of anthocyanin synthetic genes but by the absence of flavones.

\section{Effect of cyanidin}

In black cultivars, cyanidin amounts were higher than in purple cultivars (Fig. 3). Indeed, a negative correlation was observed between $L^{*}$ values and cyanidin amounts $(r=-0.689)$, indicating that cyanidin amounts also contribute to black appearance. The purple cultivars 'Cupid' and 'Atom' had lower total anthocyanidin amounts and higher cyanidin amounts than the other three cultivars (Fig. 3). Especially, despite harboring lower amounts of total anthocyanidins than 'Super Girl', 'Yukino' and 'Evelyn Rumbold', 'Cupid' had the lowest L* value of the purple cultivars (Fig. 2, Table S2), indicating that the cyanidin amount contributes to low lightness. The comparison between 'Fidalgo Blacky' and 'Black Cat', which accumulated nearly the same amounts of total anthocyanidins, supports this hypothesis. The L* value is lower in 'Fidalgo Blacky', which accumulated a higher cyanidin amount, than in 'Black Cat', which accumulated a lower cyanidin amount (Fig. 2, 3). The flowers of 'Cupid' were purple despite high amounts of cyanidin, indicating that high accumulation of cyanidin cannot alone explain the difference between black and purple cultivars. Thus, from these results, the absence of flavones contributes to black appearance through the increase of both cyanidin amounts and total anthocyanidins. To demonstrate this hypothesis, a comparison between 'Kokucho' original and the mutant was performed.

\section{Comparison between 'Kokucho' original and the mutant}

There are some reports of artificial silencing of $F N S$ and flavonol synthase $(F L S)$. In many cases, flower colors became redder (Davies et al. 2003, Holton et al. 1993, Nielsen et al. 2002), but the effect on anthocyanin amounts depended on the individual case. Silencing FNS or FLS led to increases in anthocyanins (Davies et al. 2003, Holton et al. 1993), no increase in anthocyanins (Nielsen et al. 2002), or decrease in anthocyanins (Mahajan et al. 2011, Ueyama et al. 2002). Artificial silencing occasionally 
leads to unexpected effects; hence, the effect of endogenous silencing of a lateral pathway on anthocyanin accumulation remains unclear.

The 'Kokucho' mutant produces purple flowers (Fig. 1E) with higher $\mathrm{L}^{*}$ value than the original (Fig. 2). The mutant accumulated flavones in the petals (Fig. 4B), DvFNS was expressed (Fig. 5) and no siRNA was detected (Fig. 6). Thus the comparison between the original 'Kokucho' and the mutant provides perfect conditions for investigating the relationship between DvFNS suppression and the black appearance. Although the mutant accumulated flavones in addition to anthocyanidins, the total amount of flavonoids was almost the same in the original and mutant (Fig. 4A, B). This suggests that there is a competition between the synthesis of anthocyanidins and flavones. In this scheme, the suppression of DvFNS abolished the competition between anthocyanidin and flavone synthesis, resulting in high accumulation of total anthocyanidins. How does the abolition of competition occur? At stage 3, significant differences were detected in cyanidin, apigenin, and luteolin amounts, whereas no significant difference in pelargonidin was detected (Fig. 4C). This observation indicates that an increase of cyanidin has a much greater impact on black appearance than that of pelargonidin. At stage 3, the increase of cyanidin and pelargonidin in the original and mutant were nearly identical, but because the primary amounts vary greatly between cyanidin and pelargonidin, the effect of the increase is much higher in cyanidin than in pelargonidin. It is proposed that cyanidin has a larger effect on lowering lightness than pelargonidin, but the chemical basis of this difference in effect is unknown.

To summarize these results, both total anthocyanidin amounts and cyanidin amounts are important factors in producing black appearance by reducing lightness. When flavone synthase is silenced, the competition between anthocyanidin synthesis and flavone synthesis is disrupted, leading to high accumulation of anthocyanidins. Cyanidin amounts also increase with reducing lightness, resulting in the black appearance. Finally, we present a model for black appearance (Fig. 8).

$D v F N S$ is post-transcriptionally silenced in black cultivars

Post-transcriptional gene silencing via siRNAs is one of the common mechanisms of gene silencing in many plant and animal species. In general, double-strand RNAs generated by some mechanism are processed into small (21-26 nt) RNAs, which are incorporated into an RNA-induced silencing complex composed of Argonaute protein and a small guide RNA (Ghildiyal and Zamore 2009). The 21-22-nt small RNAs typically guide mRNA cleavage, while 24-26 nt small RNAs are associated with chromatin modification or DNA methylation (Hamilton et al. 2002).

DvFNS siRNAs were detected in abundance in 'Kokucho' and 'Black Cat' and at low levels in 'Fidalgo Blacky' and 'Ms. Noir' but not in purple cultivars or the 'Kokucho' mutant line (Fig. 6). This result was consistent with the results of real-time RT-PCR, showing downregulation of $D v F N S$ in black cultivars (Fig. 5). In addition, small RNAs from 'Kokucho' were mapped onto the DvFNS genome (Fig. 
7) demonstrating that $D v F N S$ is silenced post-transcriptionally in 'Kokucho'. The observation that small RNAs from white areas of 'Yuino' petals (Ohno et al. 2011c), where DvFNS was expressed, rarely mapped to the DvFNS genome (data not shown), also supports an inference that PTGS of DvFNS occurred only in black cultivars, at least for 'Kokucho' and 'Black Cat'.

Interestingly, almost all small RNAs mapped to exon 2 of the DvFNS genome (Fig. 7). This result echoes the case of $C H S$, where small RNAs mapped to exon 2 whether PTGS occurred naturally or was artificially induced (De Paoli et al. 2009, Kurauchi et al. 2009, Morita et al. 2012, Ohno et al. 2011c, Tuteja et al. 2009). In addition, a specific 21-nt distinct antisense-oriented small RNA accounted for approximately $40 \%$ of the total mapped small RNAs. A very similar phenomenon was observed in petunia in which PhCHS-A was co-suppressed (De Paoli et al. 2009). These results suggest common mechanisms for degrading mRNA.

There are reports on naturally occurring PTGS in CHS, but not FNS. Thus, to the best of our knowledge, this is the first report on endogenous silencing of FNS. In conclusion, PTGS of FNS abolishes the competition between anthocyanin and flavone synthesis, thereby decreasing petal lightness via high accumulation of total anthocyanidins, especially cyanidin. This PTGS apparently occurs in whole petals and is relatively stable in contrast to $C H S$. Thus, PTGS of $D v F N S$ has been used as an important factor in the establishment of black cultivars in dahlia.

\section{Acknowledgments}

We thank Yukihiro Fukuda for providing the 'Kokucho' mutant line.

\section{Supplementary data}

Table S1 Primers newly designed in this experiment

Table S2 Petal colors described with CIE L*a*b*c* coordinates and flavonoid aglycon amounts for cultivars used in this experiment

Fig. S1 Relative expressions of the genes involved in anthocyanin synthesis and vacuolar sorting

Fig. S2 Relative $D v F N S$ expressions during petal development by real-time RT-PCR analysis

\section{Figure legends}


Fig. 1 Photographs of dahlia cultivars and the mutant of 'Kokucho' used in this experiment. a: 'Fidalgo Blacky’, b: ‘Ms. Noir', c: ‘Kokucho', d: ‘Black Cat', e: 'Kokucho’ mutant, f: ‘Cupid', g: 'Yukino', h: 'Super Girl', i: 'Atom', and j: 'Evelyn Rumbold'. These photographs were taken under the same incandescent light conditions. Upper line (a-d) are black cultivars: Blackness is deepest in a: Fidalgo Blacky, and the petals appear dark purple on the right side. Lower tier $(\mathrm{f}-\mathrm{j})$ are purple cultivars, with the color deeper to the left side (f). The color of 'Kokucho' mutant (e) is not black and is similar to that of the purple cultivars

Fig. 2 Distribution of $\mathrm{L}^{*}$ (lightness) and $\mathrm{c}^{*}$ (chroma) values of black and purple cultivars and the 'Kokucho' mutant.

Squares, triangles, and the circle indicate black cultivars, purple cultivars, and the 'Kokucho' mutant, respectively. Each data represents the average of total 27 collected datapoints: 3 locations $\times 3$ petals $\times 3$ independent flowers. The values of $\mathrm{L}^{*}$ were lower in black cultivars (14.1-15.9) than in purple cultivars (20.6-29.5). The values of $\mathrm{L}^{*}$ and $\mathrm{c}^{*}$ in the 'Kokucho' mutant were 17.2 and 119.9, respectively, which were higher than those of the original cultivar, 'Kokucho' $\left(\mathrm{L}^{*}=14.9, \mathrm{c}^{*}=94.2\right)$. The raw data are shown in supplemental data Table S2

Fig. 3 Contents of flavonoid aglycones in petals of black and purple cultivars.

Lt: luteolin, Ap: apigenin, Cy: cyanidin, and Pg: pelargonidin. All data represent the average \pm SE of each flavonoid (three biological replications; the data of 'Cupid' represent two biological replications). Stage 4 completely colored and expanded petals were used. The raw data are shown in supplemental data Table S2. The data for the 'Kokucho' mutant are shown in Fig. 4

Fig. 4 Contents of flavonoid aglycones in petals at four developmental stages. a: 'Kokucho' original cultivar, b: 'Kokucho' mutant. c: The amounts of each aglycone in the 'Kokucho' original cultivar and mutant at stage 3. Lt: luteolin, Ap: apigenin, Cy: cyanidin, and Pg: pelargonidin. Four petal developmental stages; stage 1 (S1): uncolored, stage 2 (S2): half colored, stage 3 (S3): completely colored and unexpanded, stage 4 (S4): completely colored and expanded. All data represent average \pm SE (three biological replications). Statistical analysis was performed with the Mann-Whitney $U$ test. *: significant at $P<0.05$, N.S.: not significant

Fig. 5 Relative $D v F N S$ expressions in petals by real-time RT-PCR analysis. RNAs were extracted from stage 2 half-colored petals. All data represent average \pm SE (three biological replications). Each data represents the expression level relative to that of 'Yukino'. DvActin was used as the internal standard. Calculation was conducted using a standard curve prepared from a diluted series of 'Yukino' cDNA 
Fig. 6 RNA gel blot hybridization for $D v F N S$ siRNA detection.

1: 'Fidalgo Blacky', 2: 'Ms. Noir', 3: 'Black Cat', 4: 'Kokucho', 5: 'Kokucho' mutant, 6: 'Super Girl', 7:

'Yukino', 8: 'Cupid', 9: 'Evelyn Rumbold', and 10: 'Atom'. siRNA were detected only in black cultivars.

Ribosome RNA on the electrophoresed gel is shown in the lower photograph

Fig. 7 Mapping of small RNAs on DvFNS genome. Small RNAs (18-32 nt long) without mismatch to 'Kokucho' DvFNS DNA were mapped on either the sense strand (pink: above the X-axis) or the antisense strand (blue: below the Y-axis)

Fig. 8 A model for black flower production.

(a) When FNS is suppressed, the competition between synthesizing flavones and anthocyanidins is abolished. The substrates used for flavone synthesis are then available for synthesis of anthocyanidins. Thus, anthocyanidin concentrations increase in a FNS suppressed flower relative to those in a non-suppressed flower.

(b) From the results of Figs. 2, 3, and 4, it is considered that cyanidin molecules will contribute much more to lowering lightness than the same number of pelargonidin molecules. We define "darkness unit" to explain the black appearance, which indicates the contribution of a molecule to lowering lightness. When the sum of darkness unit approaches the hypothetical threshold, flower color looks black to the eye. For example, the darkness unit of cyanidin is considered to be twice that of pelargonidin based on the report (Saito and Mikanagi 1989). Four representative cases are shown. (1) Total anthocyanidins exceed the hypothetical threshold. (2) Cyanidin concentrations lead to exceeding the hypothetical threshold. (3) and (4) The hypothetical threshold is not exceeded. White: darkness unit of pelargonidin; gray: darkness unit of original cyanidin, and black: darkness unit of increased cyanidin

Fig. S1 Relative expressions of the genes involved in anthocyanin synthesis and vacuolar sorting. a: $D v I V S, \mathbf{b}: D v C H S 1, \mathbf{c}: D v C H S 2, \mathbf{d}: D v C H I, \mathbf{e}: D v F 3 H, \mathbf{f}: D v D F R, \mathbf{g}: D v A N S, \mathbf{h}: D v 3 G T, \mathbf{i}: D v 3 M T, \mathbf{j}:$ $D v G S T, \mathbf{k}: D v F 3^{\prime} H$.

RNAs were extracted from stage 3, completely colored and unexpanded petals. For $D v F 3$ ' $H$, RNAs extracted from stage 2 half-colored petals were used. All data represent as the average \pm SE (three biological replications; The data of 'Kokucho' mutant is from five biological replications). Each data represents the expression level relative to the maximum data (DvIVS, DvCHS2: 'Kokucho' mutant, DvCHS1, DvF3H, DvDFR, DvANS, Dv3GT, Dv3MT: 'Yukino', DvCHI: 'Kokucho', DvGST, DvF3'H: 'Black Cat'). DvActin was used as the internal standard. Calculation was conducted using the standard curve prepared from the diluted series of 'Yukino' cDNA 
Fig. S2 Relative $D v F N S$ expressions during petal development by real-time RT-PCR analysis. RNAs were extracted from petals at three developmental stages: stage 1 (S1): uncolored, stage 2 (S2): half colored, and stage 3 (S3): completely colored and unexpanded. Each data represents the expression level relative to that of 'Yukino' stage 2. DvActin was used as an internal standard. Calculation was based on a standard curve prepared from a diluted series of 'Yukino' cDNA

\section{Reference}

Akashi T, Fukuchi-Mizutani M, Aoki T, Ueyama Y, Yonekura-Sakakibara K, Tanaka Y, Kusumi T, Ayabe SI (1999) Molecular cloning and biochemical characterization of a novel cytochrome P450, flavone synthase II, that catalyzes direct conversion of flavanones to flavones. Plant Cell Physiol 40:1182-1186

Azuma A, Udo Y, Sato A, Mitani N, Kono A, Ban Y, Yakushiji H, Koshita Y, Kobayashi S (2011) Haplotype composition at the color locus is a major genetic determinant of skin color variation in Vitis $\times$ labruscana grapes. Theor Appl Genet 122:1427-1438

Davies KM, Schwinn KE, Deroles SC, Manson DG, Lewis DH, Bloor SJ, Bradley JM (2003) Enhancing anthocyanin production by altering competition for substrate between flavonol synthase and dihydroflavonol 4-reductase. Euphytica 131:259-268

De Paoli E, Dorantes-Acosta A, Jixian Z, Accerbi M, Jeong DH, Sunhee P, Meyers BC, Jorgensen RA, Green PJ (2009) Distinct extremely abundant siRNAs associated with cosuppression in petunia. RNA 15:1965-1970

Fliegmann J, Furtwängler K, Malterer G, Cantarello C, Schüler G, Ebel J, Mithöfer A (2010) Flavone synthase II (CYP93B16) from soybean (Glycine max L.). Phytochemistry 71:508-514

Gatt M, Ding H, Hammett K, Murray B (1998) Polyploidy and evolution in wild and cultivated Dahlia species. Ann Bot 81:647-656

Ghildiyal M, Zamore PD (2009) Small silencing RNAs: An expanding universe. Nat Rev Gen 10:94-108 Grotewold E (2006) The genetics and biochemistry of floral pigments. Annu Rev Plant Biol 57:761-780 
Hamilton A, Voinnet O, Chappell L, Baulcombe D (2002) Two classes of short interfering RNA in RNA silencing. EMBO J 21:4671-4679

Harborne JB, Greenham J, Eagles J (1990) Malonylated chalcone glycosides in Dahlia. Phytochemistry 29:2899-2900

Hichri I, Barrieu F, Bogs J, Kappel C, Delrot S, Lauvergeat V (2011) Recent advances in the transcriptional regulation of the flavonoid biosynthetic pathway. J Exp Bot 62:2465-2483

Holton TA, Brugliera F, Tanaka Y (1993) Cloning and expression of flavonol synthase from Petunia hybrida. Plant J 4:1003-1010

Kitamura S, Shikazono N, Tanaka A (2004) TRANSPARENT TESTA 19 is involved in the accumulation of both anthocyanins and proanthocyanidins in Arabidopsis. Plant J 37:104-114

Kobayashi S (2009) Regulation of anthocyanin biosynthesis in grapes. J Jpn Soc Hortic Sci 78:387-393

Kobayashi S, Goto-Yamamoto N, Hirochika H (2005) Association of VvmybAl gene expression with anthocyanin production in grape (Vitis vinifera) skin-color mutants. J Jpn Soc Hortic Sci 74:196-203

Kobayashi S, Ishimaru M, Hiraoka K, Honda C (2002) Myb-related genes of the Kyoho grape (Vitis labruscana) regulate anthocyanin biosynthesis. Planta 215:924-933

Koes R, Verweij W, Quattrocchio F (2005) Flavonoids: A colorful model for the regulation and evolution of biochemical pathways. Trends Plant Sci 10:236-242

Kurauchi T, Matsumoto T, Taneda A, Sano T, Senda M (2009) Endogenous short interfering RNAs of chalcone synthase genes associated with inhibition of seed coat pigmentation in soybean. Breed Sci 59:419-426

Lawrence WJC, Scott-Honcrieff R (1935) The genetics and chemistry of flower colour in Dahlia: A new theory of specific pigmentation. J Genet 30:155-226

Lawrence WJC (1931) The genetics and cytology of Dahlia variabilis. J Genet 24:257-306

Lawrence WJC (1929) The genetics and cytology of Dahlia species. J Genet 21:125-159

Mahajan M, Ahuja PS, Yadav SK (2011) Post-transcriptional silencing of flavonol synthase mRNA in tobacco leads to fruits with arrested seed set. PLoS ONE 6: e28315 
Martens S, Mithöfer A (2005) Flavones and flavone synthases. Phytochemistry 66:2399-2407

Martens S, Forkmann G (1999) Cloning and expression of flavone synthase II from Gerbera hybrids. Plant J 20:611-618

Martens S, Forkmann G (1998) Genetic control of flavone synthase II activity in flowers of Gerbera hybrids. Phytochemistry 49:1953-1958

McClaren M. 2009. Dahlia: history and species. In: McClaren B, Encyclopedia of dahlias. Timber Press, Portland, pp161-166

Morita Y, Saito R, Ban Y, Tanikawa N, Kuchitsu K, Ando T, Yoshikawa M, Habu Y, Ozeki Y, Nakayama M (2012) Tandemly arranged chalcone synthase A genes contribute to the spatially regulated expression of siRNA and the natural bicolor floral phenotype in Petunia hybrida. Plant J 70:739-749

Mueller LA, Goodman CD, Silady RA, Walbot V (2000) AN9, a petunia glutathione S-transferase required for anthocyanin sequestration, is a flavonoid-binding protein. Plant Physiol 123:1561-1570

Nakatsuka T, Nishihara M, Mishiba K, Yamamura S (2005) Temporal expression of flavonoid biosynthesis-related genes regulates flower pigmentation in gentian plants. Plant Sci 168:1309-1318

Nesi N, Debeaujon I, Jond C, Pelletier G, Caboche M, Lepiniec L (2000) The TT8 gene encodes a basic helix-loop-helix domain protein required for expression of $D F R$ and $B A N$ genes in Arabidopsis siliques. Plant Cell 12:1863-1878

Nielsen K, Deroles SC, Markham KR, Bradley MJ, Podivinsky E, Manson D (2002) Antisense flavonol synthase alters copigmentation and flower color in lisianthus. Mol Breed 9:217-229

Nordström CG, Swain T (1953) The flavonoid glycosides of Dahlia variabilis. Part I. General introduction. cyanidin, apigenin, and luteolin glycosides from the variety "Dandy". J Chem Soc:2764-2773

Ohno S, Hosokawa M, Hoshino A, Kitamura Y, Morita Y, Park KI, Nakashima A, Deguchi A, Tatsuzawa F, Doi M, Iida S, Yazawa S (2011a) A bHLH transcription factor, DvIVS, is involved in regulation of anthocyanin synthesis in dahlia (Dahlia variabilis). J Exp Bot 62:5105-5116

Ohno S, Deguchi A, Hosokawa M, Tatsuzawa F, Doi M (2011b) Accumulation of flavonoid pigments characteristic of blackish Dahlia petals. Hort Res 10 (Suppl. 2): 564 (In Japanese). 
Ohno S, Hosokawa M, Kojima M, Kitamura Y, Hoshino A, Tatsuzawa F, Doi M, Yazawa S (2011c) Simultaneous post-transcriptional gene silencing of two different chalcone synthase genes resulting in pure white flowers in the octoploid dahlia. Planta 234:945-958

Price JR (1939) The yellow colouring matter of Dahlia variabilis. J Chem Soc:1017-1018

Saito N, Mikanagi Y (1989) Sekishokushikiso to sekishokuka. Bio Horti 3: 49-62 (In Japanese)

Spelt C, Quattrocchio F, Mol JNM, Koes R (2000) anthocyaninl of petunia encodes a basic helix-loop-helix protein that directly activates transcription of structural anthocyanin genes. Plant Cell $12: 1619-1631$

Takeda K, Harborne JB, Self R (1986) Identification and distribution of malonated anthocyanins in plants of the compositae. Phytochemistry 25:1337-1342

Tanaka Y, Sasaki N, Ohmiya A (2008) Biosynthesis of plant pigments: Anthocyanins, betalains and carotenoids. Plant J 54:733-749

Thill J, Miosic S, Ahmed R, Schlangen K, Muster G, Stich K, Halbwirth H (2012) 'Le Rouge et le Noir': A decline in flavone formation correlates with the rare color of black dahlia (Dahlia variabilis hort.) flowers. BMC Plant Biol: 225

Tuteja JH, Zabala G, Varala K, Hudson M, Vodkin LO (2009) Endogenous, tissue-specific short interfering RNAs silence the chalcone synthase gene family in Glycine max seed coats. Plant Cell 21:3063-3077

Ueyama Y, Suzuki KI, Fukuchi-Mizutani M, Fukui Y, Miyazaki K, Ohkawa H, Kusumi T, Tanaka Y (2002) Molecular and biochemical characterization of torenia flavonoid 3'-hydroxylase and flavone synthase II and modification of flower color by modulating the expression of these genes. Plant Sci $163: 253-263$ 


\section{Figures}

Fig.1

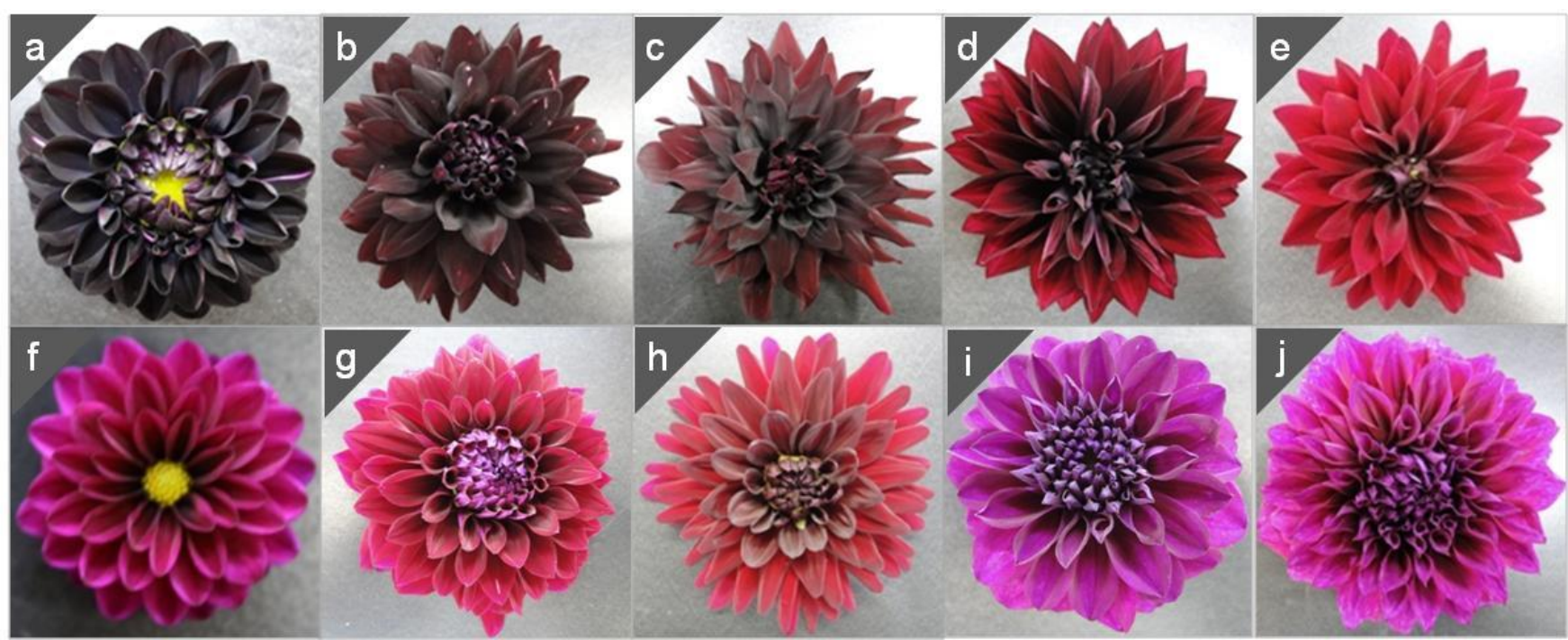


Fig. 2

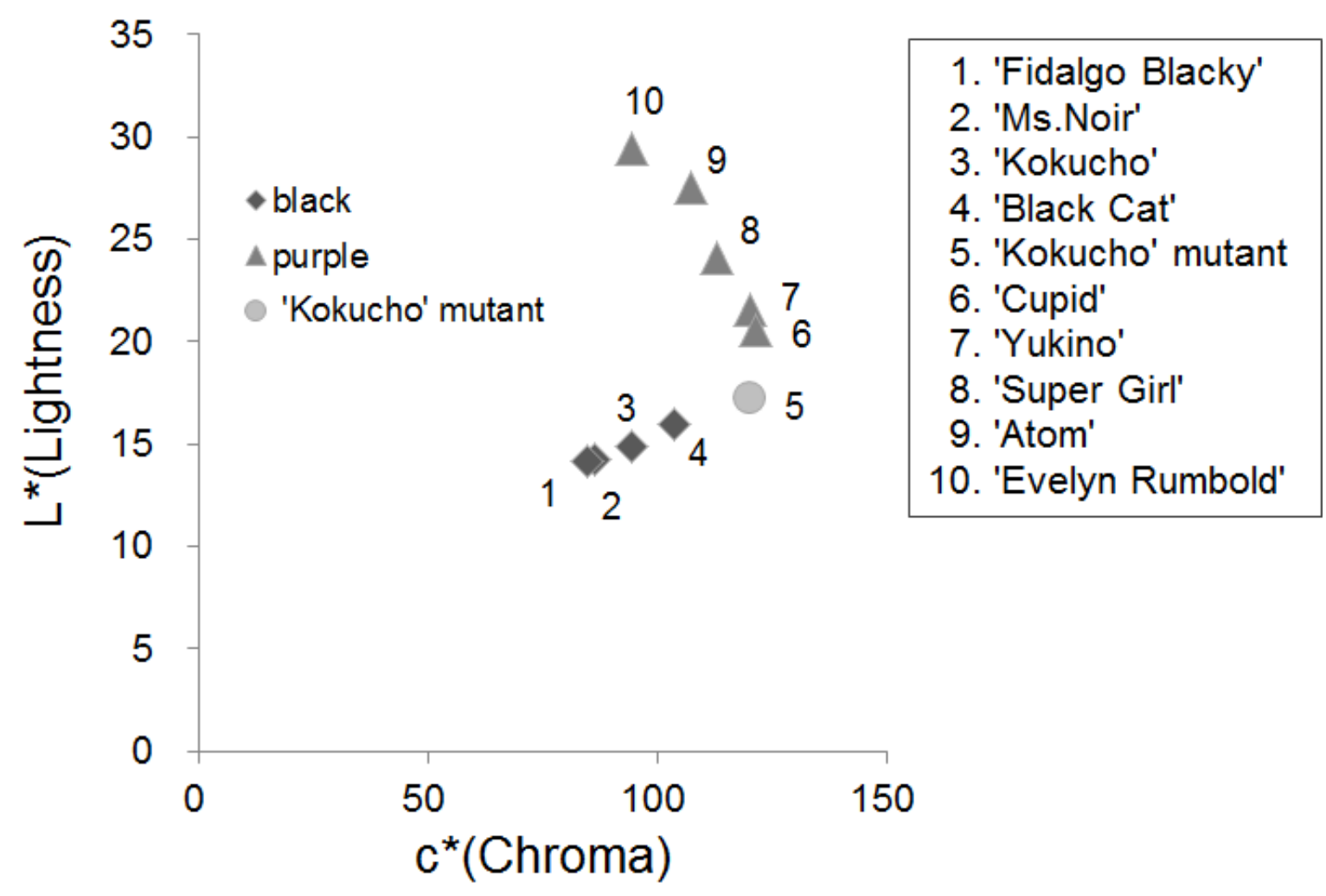


Fig. 3

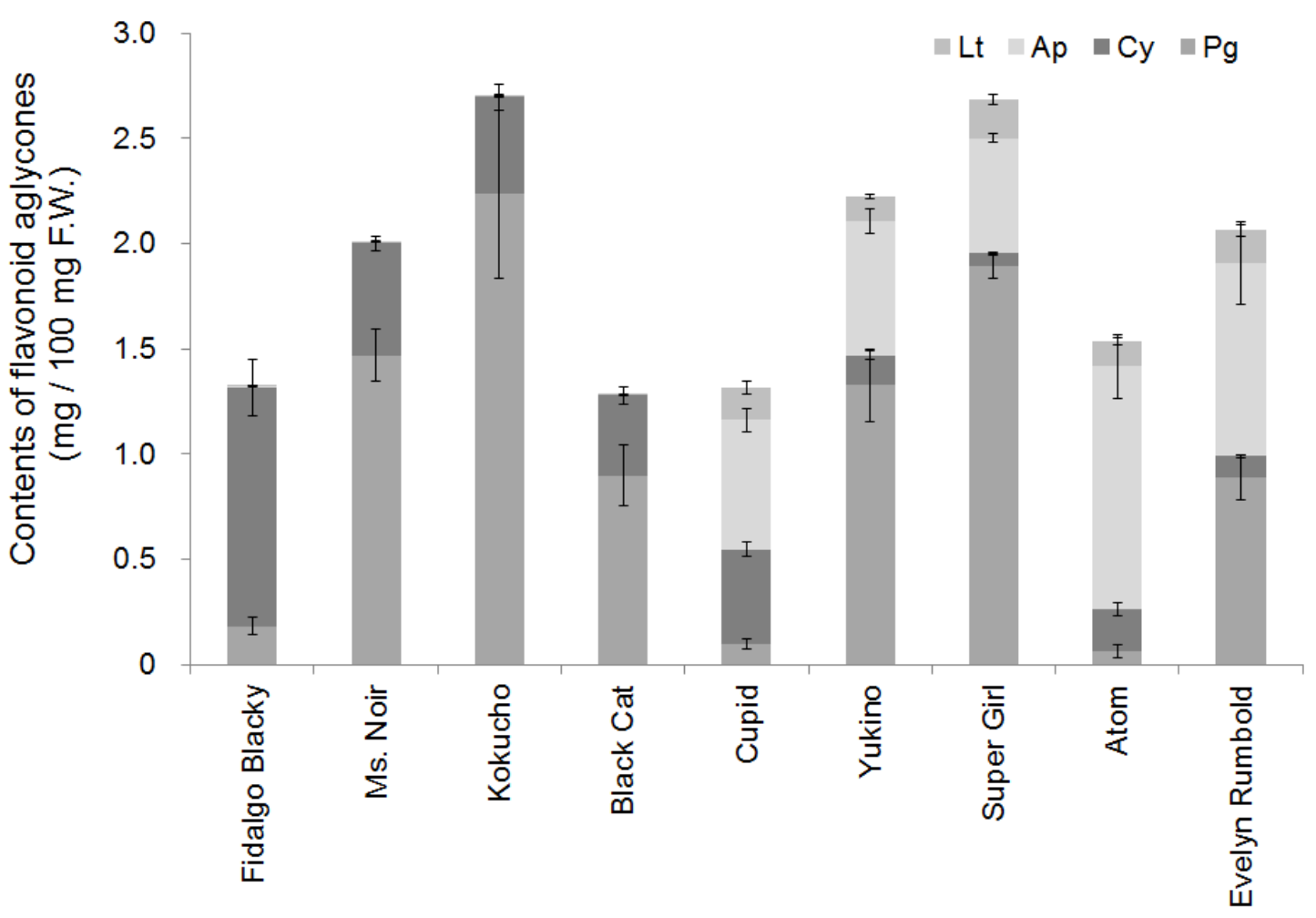


Fig. 4

a

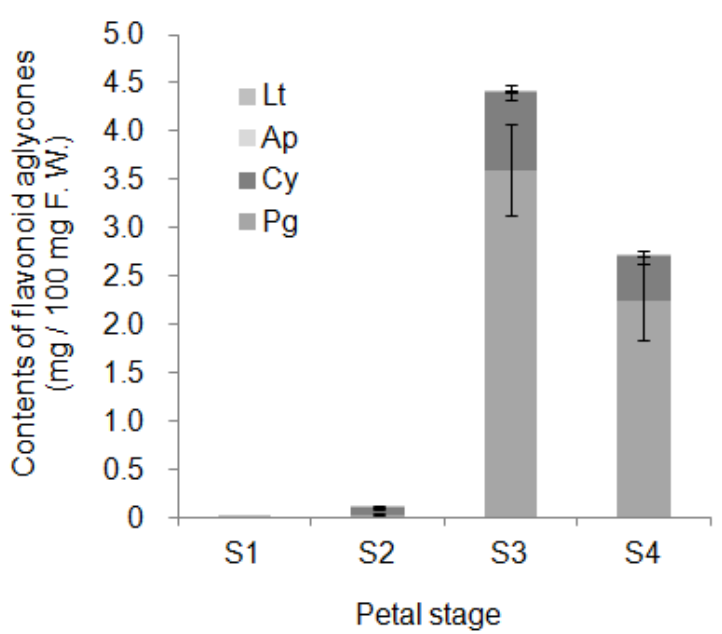

C

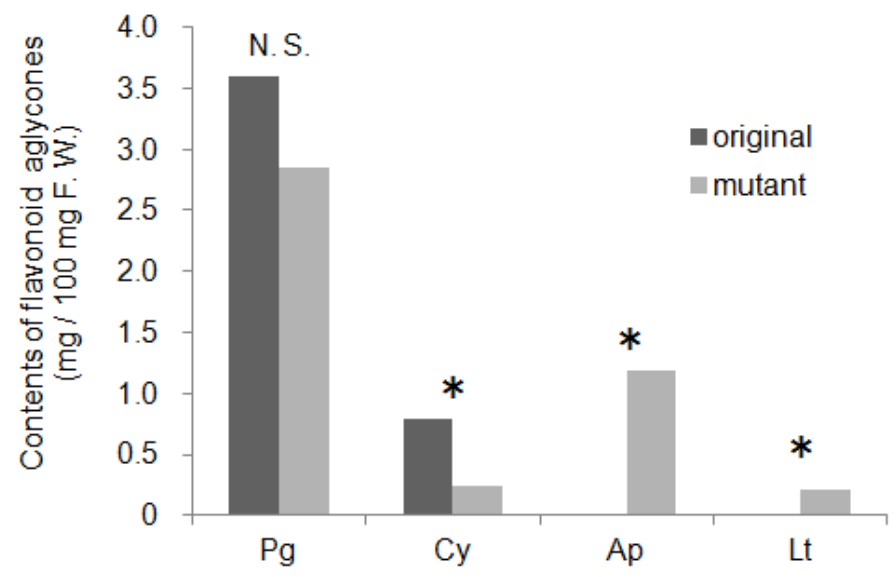

b

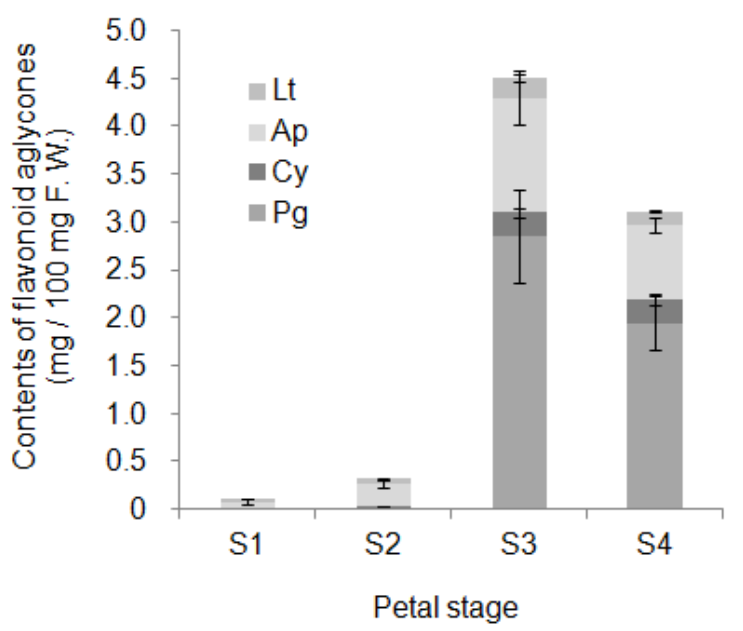


Fig.5

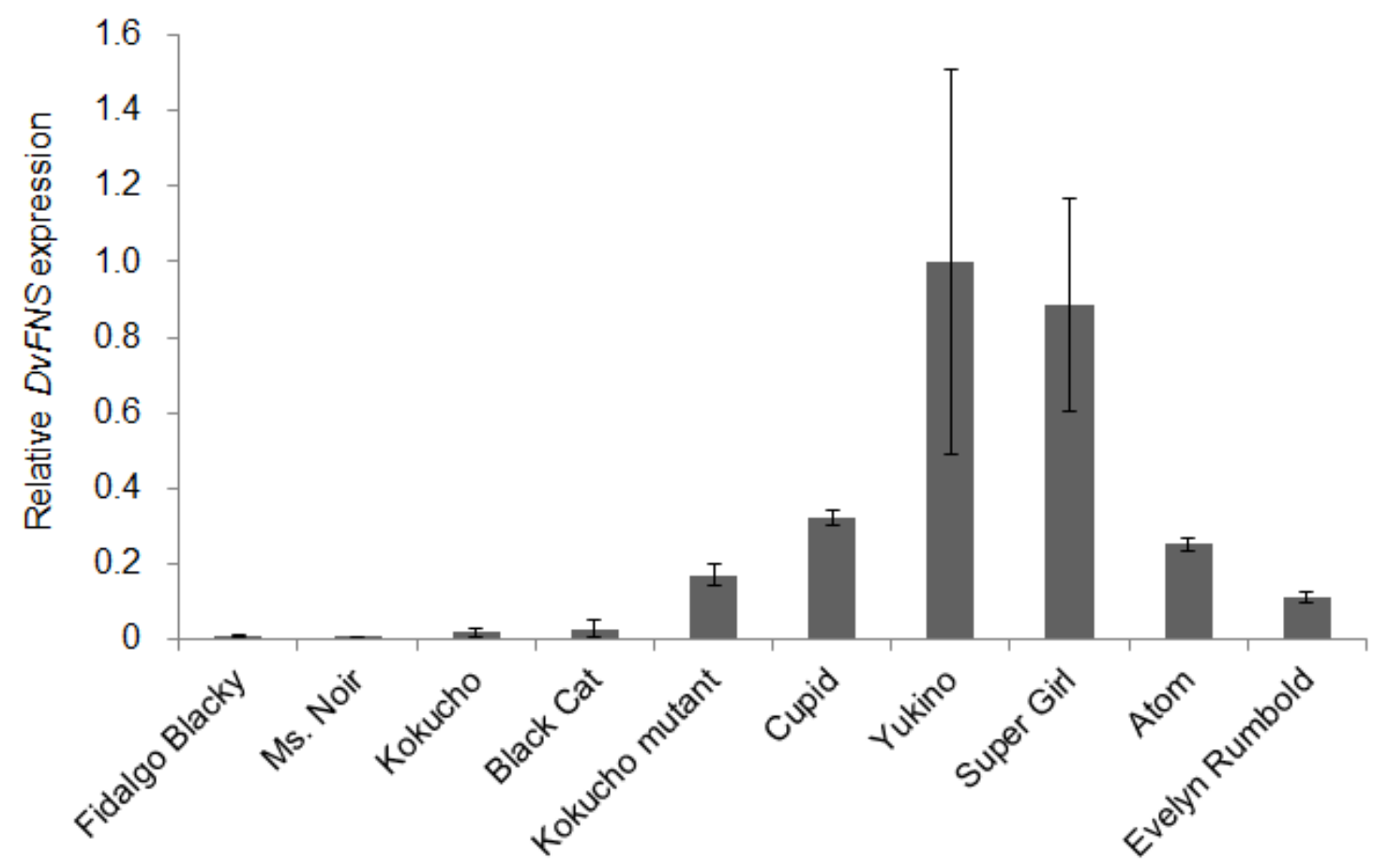


Fig. 6

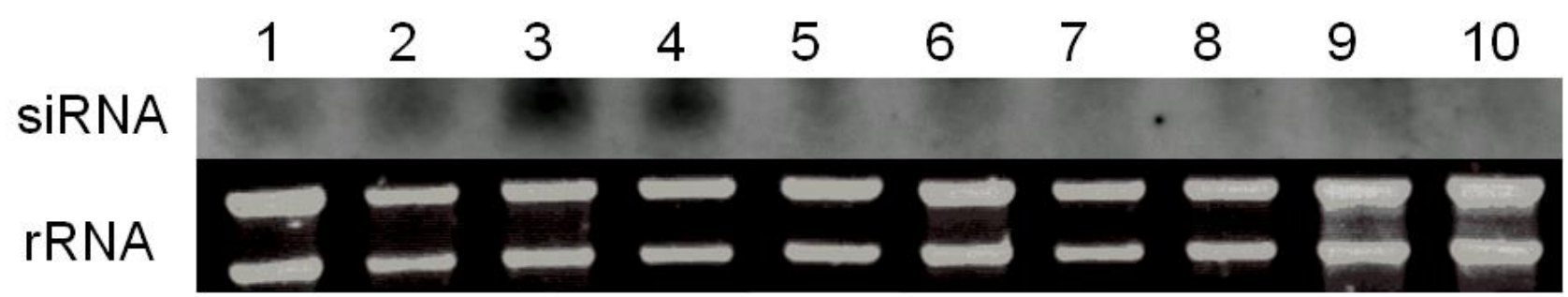


Fig. 7

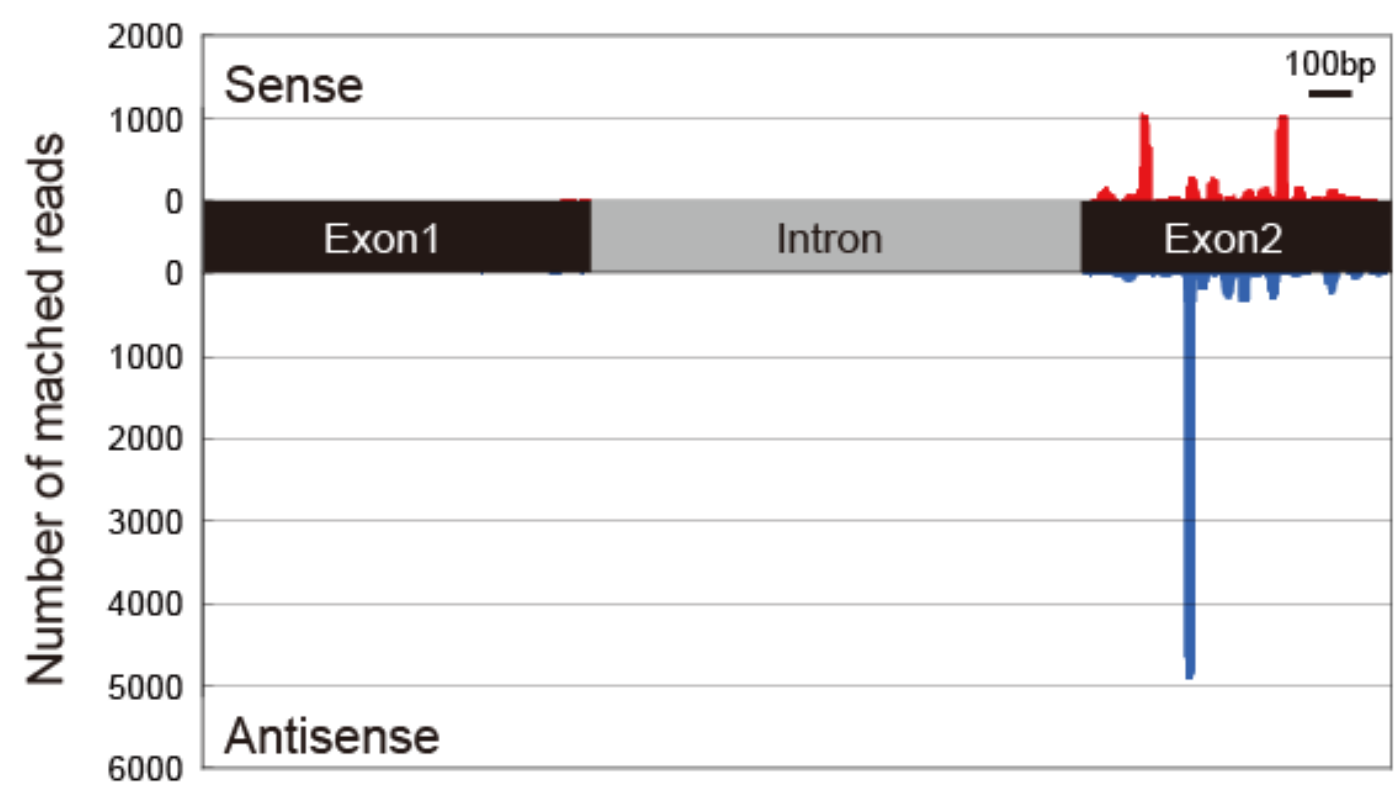


Fig. 8

(a)

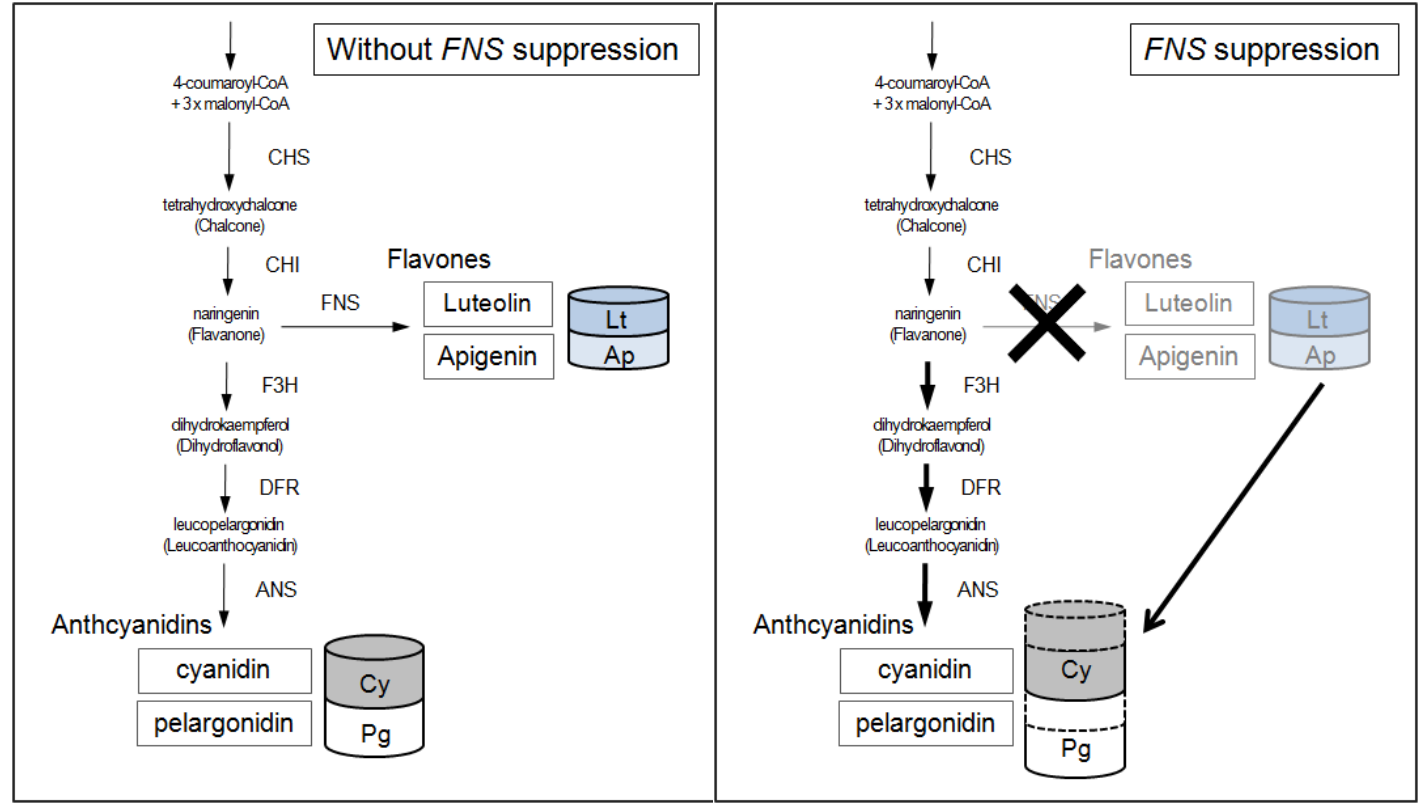

(b)

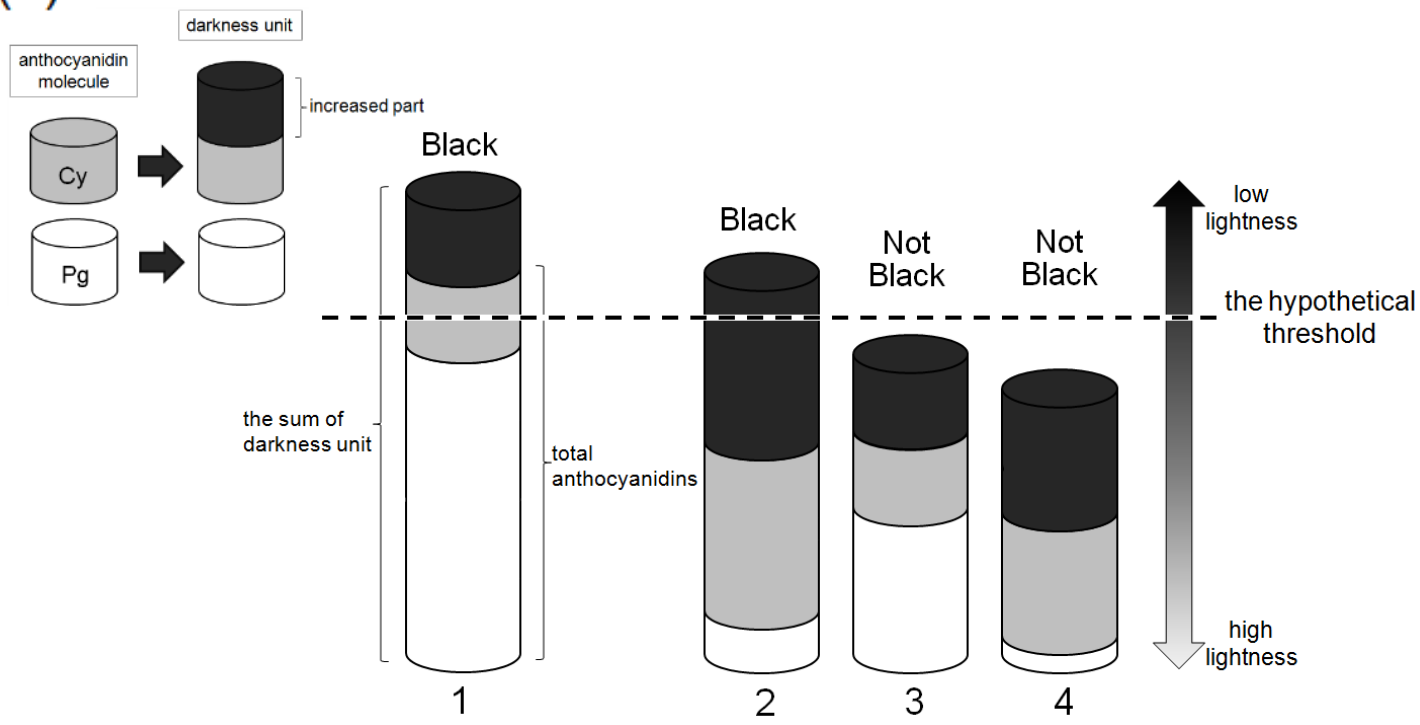




\section{Supplementary data}

Table S1 Primers newly designed in this experiment

\begin{tabular}{|c|c|c|c|c|}
\hline Gene & Purpose & Name & Orientation & sequence $\left(5^{\prime}-3^{\prime}\right)$ \\
\hline \multirow{2}{*}{ DV3MT } & Real-time & $\mathrm{F}$ & sense & AAATACGAAGTTGTTTCAATC \\
\hline & Real-time & $\mathrm{R}$ & antisense & TTGCACTTTCTAATCCATCAT \\
\hline \multirow{22}{*}{ DvFNS } & Degenerate & deg-FNS-F & sense & YCACATYAARGCMYTGRTTTTGGA \\
\hline & Degenerate & deg-FNS-R & antisense & TDGACCAAATRTTBACRAAYAACA \\
\hline & Race & FNS 3'Race & sense & GCTGGAACAGACACAACCGCAGTA \\
\hline & Race & FNS 3'Race-1 & sense & ATGTTATTCGTAAACATTT \\
\hline & Race & FNS 3'Race-1R & antisense & ACCTCGTTCATCCATATT \\
\hline & Race & FNS 3'Race-2F & sense & AATATGGATGAACGAGGT \\
\hline & Race & FNS 3'Race-nested & sense & CССTATGGTCATGGAAAAAGCAAA \\
\hline & Race & FNS 5'Race & antisense & СТСАСАТTTTСАТTTGАСTTCCGTA \\
\hline & Race & FNS 5'Race-1F & antisense & AATCCACCTCCGTCTCGGTT \\
\hline & Race & FNS 5'Race-1R & antisense & ATATCCTCGTACCTCTTCTT \\
\hline & Race & FNS 5'Race-nested & antisense & GGTGGATGAAGTCGGAAGGCTTCT \\
\hline & Real-time & FNS real-time F & sense & GTGTGTTTCCCTTTGCTTCGTAAAA \\
\hline & Real-time & FNS real-time $R$ & antisense & GCGAAGGGAAACACACTAGATTCGT \\
\hline & Sequence & FNS Full-F & sense & TCTCATCTTACCATGAATACACT \\
\hline & Sequence & FNS Full-R & antisense & GCGAAGGGAAACACACTAGATTCG \\
\hline & Sequence & FNS-1161R & antisense & ATATCCGGATTTAACACTCACA \\
\hline & Sequence & FNS-488F & sense & CAAACAAAATGAGAGTGTGAA \\
\hline & Sequence & FNS-800F & sense & ATGGCAAAGGGAAAGATTTTCTAGA \\
\hline & Sequence & FNS-942R & antisense & TATTATTACTGCGGTTGTGTCTGTT \\
\hline & Sequence & FNS-IntF1 & sense & ACGTGTGAGTTGAGCATCCCGAAT \\
\hline & Sequence & FNS-IntR1 & antisense & TAATCTTGAAATAATTAAGA \\
\hline & Sequence & FNS-IntR2 & antisense & TGTGCGTTAAAAATCTAGGGTCAT \\
\hline \multirow{7}{*}{ DVGST } & Race & GST 3'Race & sense & CCGATTAGGGCGGCTTGTCCACAAA \\
\hline & Race & GST 3'Race-1 & sense & TGTGTAAAAAAGCTTGAA \\
\hline & Race & GST 3'Race-nested & sense & CCGCGTTAACTTAGACTCCGGAGAA \\
\hline & Real-time & GST real-time F & sense & ATGTGGTGGGATGATATTTCAAACA \\
\hline & Real-time & GST real-time $R$ & antisense & AACATTTATTTGTGAGTCACATACA \\
\hline & Sequence & GST Full-F & sense & GTCCATTTAACAAGAACTTGGAGCT \\
\hline & Sequence & GST Full-R & antisense & ATAAAACATTTATTTGTGAGTCACA \\
\hline \multirow{2}{*}{$D v F 3^{\prime} H$} & Real-time & F3'H real-time F & sense & TCGGCTTCGTTGACGTGGTG \\
\hline & Real-time & F3'H real-time R & antisense & TACGGTGCAAACACCAGATCC \\
\hline
\end{tabular}
Y: C or T, R: A or G, M: A or C, D: A, G or T, B: C, G or T 
Table S2 Petal colors described with CIE L ${ }^{*} a^{*} b^{*} c^{*}$ coordinates and flavonoid aglycon amounts for cultivars used in this experiment

\begin{tabular}{ccccccccc}
\hline \multirow{2}{*}{ Cultivar } & \multicolumn{3}{c}{ CIE L ${ }^{*}{ }^{*} \mathrm{~b}^{*} \mathrm{c}^{*}$ coordinates } & \multicolumn{3}{c}{ Flavonoid aglycon amounts (mg/100mg fresh petals) } \\
\cline { 2 - 8 } & $\mathrm{L}^{*}$ & $\mathrm{a}^{*}$ & $\mathrm{~b}^{*}$ & $\mathrm{c}^{*}$ & Peralgonidin & Cyanidin & Apigenin & Luteolin \\
\hline Fidalgo Blacky & 14.14 & 84.53 & 2.34 & 84.57 & 0.18 & 1.13 & 0.01 & 0.00 \\
Ms. Noir & 14.22 & 86.01 & 3.06 & 86.07 & 1.47 & 0.53 & 0.00 & 0.01 \\
Kokucho & 14.73 & 96.82 & 4.22 & 96.91 & 2.37 & 0.45 & 0.00 & 0.00 \\
Black Cat & 15.94 & 103.53 & 6.58 & 103.75 & 0.90 & 0.38 & 0.00 & 0.00 \\
Kokucho mutant & 17.22 & 119.62 & 8.76 & 119.94 & 1.94 & 0.24 & 0.78 & 0.14 \\
Cupid & 20.55 & 121.42 & -0.96 & 121.44 & 0.10 & 0.45 & 0.62 \\
Yukino & 21.53 & 119.50 & 11.46 & 120.09 & 1.33 & 0.14 & 0.64 & 0.15 \\
Super Girl & 24.10 & 111.74 & 13.76 & 112.70 & 1.89 & 0.06 & 0.55 & 0.12 \\
Atom & 27.53 & 106.92 & -7.47 & 107.20 & 0.06 & 0.20 & 1.15 & 0.12 \\
Evelyn Rumbold & 29.45 & 94.23 & 1.39 & 94.40 & 0.89 & 0.10 & 0.92 & 0.16 \\
\hline
\end{tabular}

$c^{*}$ was culculated as $\left(a^{* 2}+b^{* 2}\right)^{1 / 2}$ 
Fig. S1

a

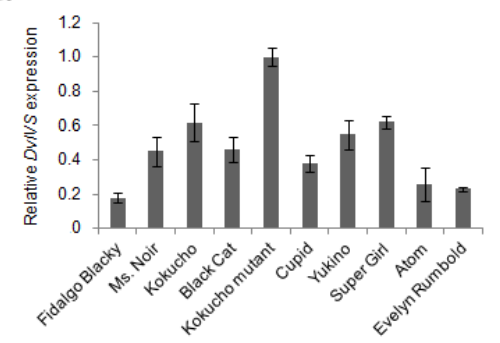

d

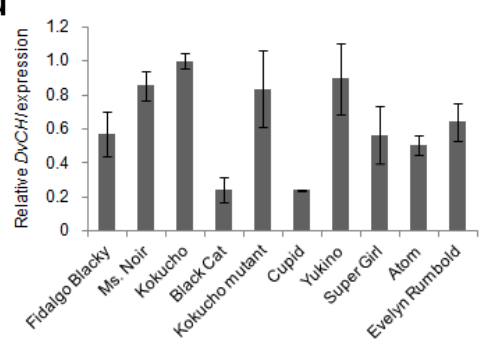

g

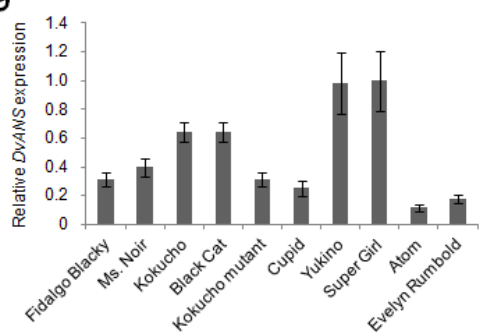

j

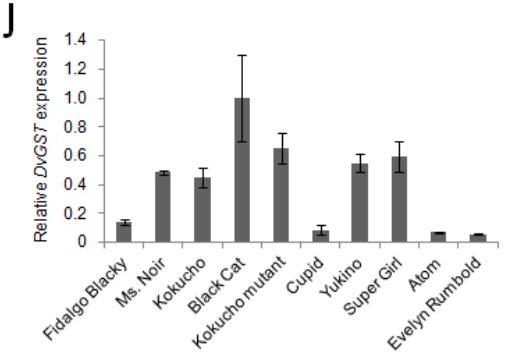

b

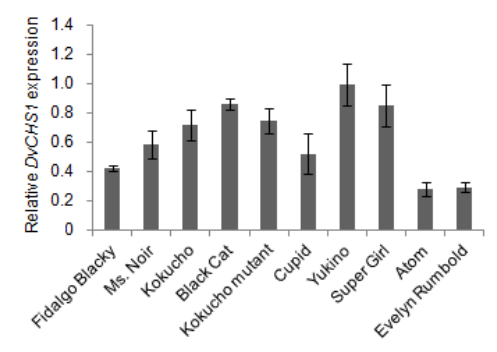

e

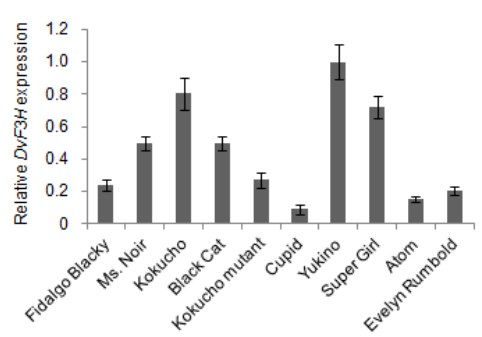

$\mathrm{h}$

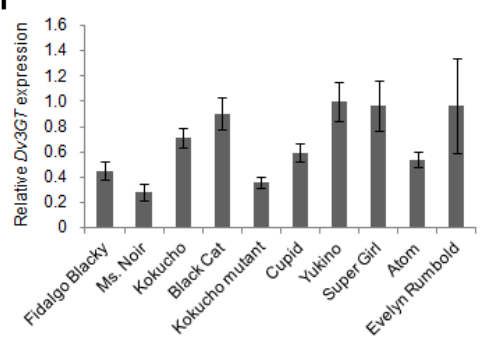

tirl
C

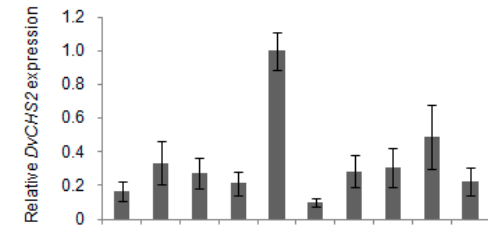

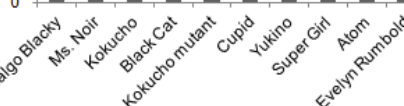

$\mathrm{f}$

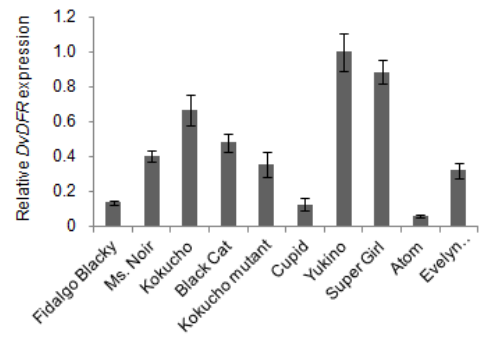

i

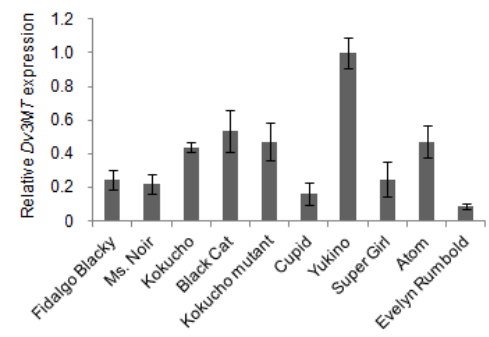


Fig. S2

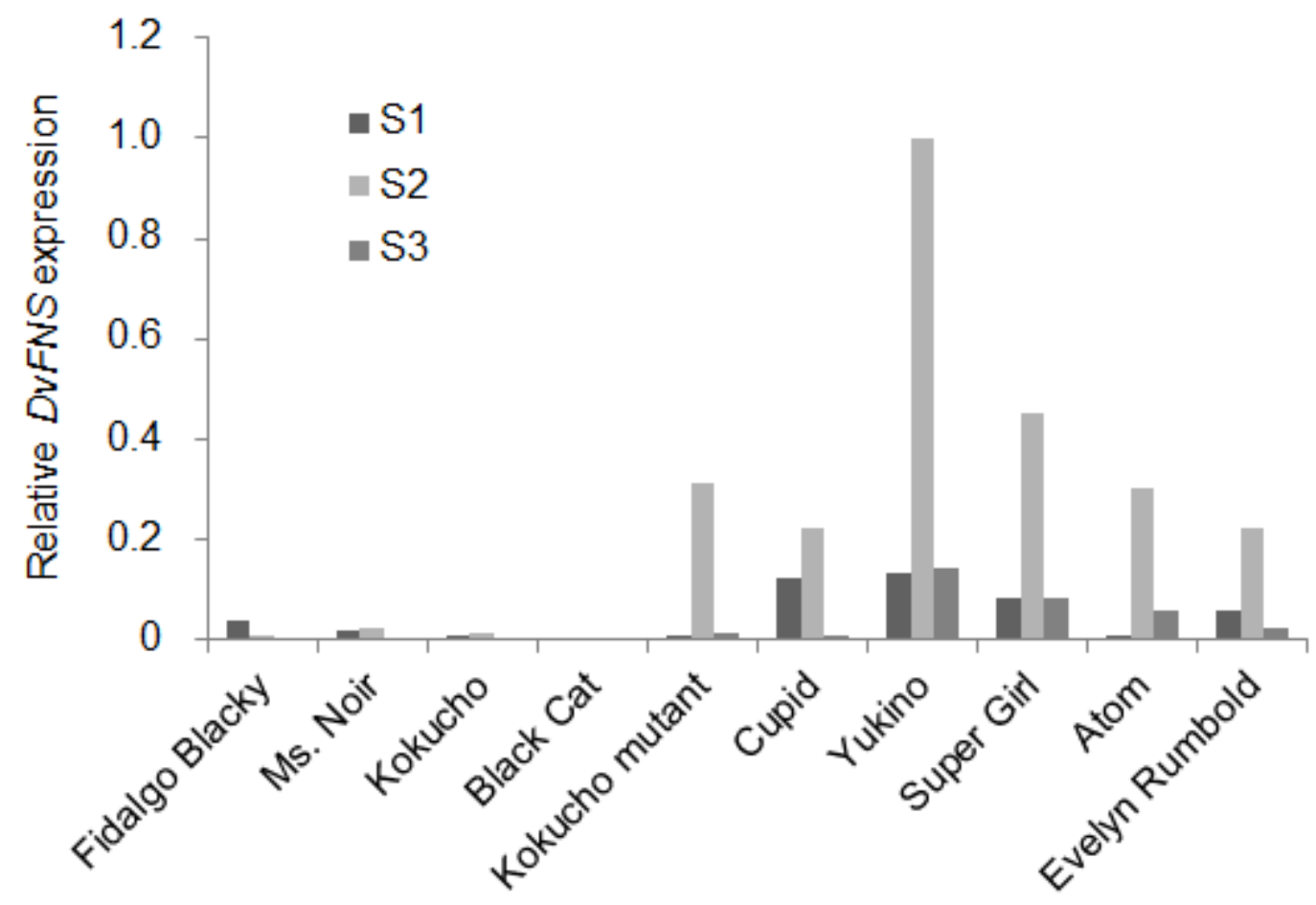

